\title{
PRESENCIA DE LAS UNIVERSIDADES ESPAÑOLAS EN LAS REDES SOCIALES DIGITALES CIENTÍFICAS: CASO DE LOS ESTUDIOS DE COMUNICACIÓN
}

\author{
Presence of Spanish universities on scientific digital \\ social networks: case of communication studies
}

\section{Cristina González-Díaz, Mar Iglesias-García y Lluís Codina}

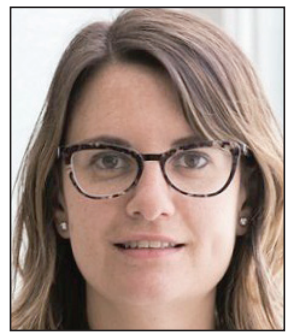

Cristina González-Díaz, licenciada en publicidad y relaciones públicas, es profesora en el Departamento de Comunicación y Psicología Social de la Universidad de Alicante e imparte docencia en el grado de publicidad y relaciones públicas. Dirige el proyecto de investigación Estudio de la presencia de los mensajes de salud en la publicidad de alimentos, financiado por la Universidad de Alicante.

http://orcid.org/0000-0001-6981-0499

Universidad de Alicante Campus Sant Vicent del Raspeig, Ap. 99. 03080 Alicante, España cristina.gdiaz@ua.es

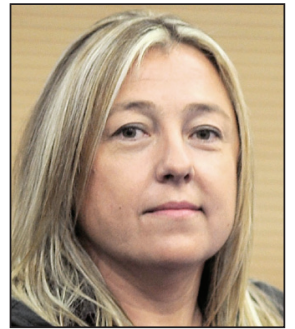

Mar Iglesias-García es periodista y profesora del Departamento de Comunicación y Psicología Social de la Universidad de Alicante. Imparte docencia en el grado de publicidad y relaciones públicas y en el grado de turismo. Colabora en el proyecto de investigación Audiencias activas y periodismo. Interactividad, integración en la web y buscabilidad de la información periodística, financiado por el Ministerio de Economía y Competitividad. Desde 2010, es directora del ciberperiódico Comunic@ndoUA.

http://orcid.org/0000-0001-7926-5746

Universidad de Alicante Campus Sant Vicent del Raspeig, Ap. 99. 03080 Alicante, España mar.iglesias@ua.es

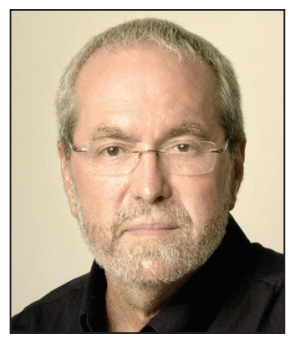

Lluís Codina es profesor de la Universitat Pompeu Fabra de Barcelona. Imparte docencia en la Facultad de Comunicación, en los grados de periodismo y de comunicación audiovisual. Es coordinador del Máster universitario en comunicación social (MUCS) del Departamento de Comunicación. Forma parte del Grupo de Investigación en Documentación Digital y Comunicación Interactiva (DigiDoc). Su último libro, en colaboración, trata sobre sistemas de evaluación de sitios web. http://orcid.org/0000-0001-7020-1631

Universitat Pompeu Fabra Roc Boronat, 138. 08018 Barcelona, España Iluis.codina@upf.edu

\section{Resumen}

Las redes sociales digitales científicas (RSDC) suponen un nuevo punto de encuentro para los investigadores y un instrumento de primer orden para la promoción del conocimiento. Actualmente su uso se está incrementando entre la comunidad científica, ya que son plataformas ágiles para difundir los resultados de las investigaciones, así como para compartir conocimiento. Este trabajo estudia la presencia de las universidades españolas en las dos principales (ResearchGate y Academia. edu), a partir del análisis del personal académico adscrito a 77 universidades españolas, públicas y privadas. Los resultados muestran que, a pesar del desarrollo que están experimentando en todo el mundo, el número de miembros españoles en estas redes sociales es insuficiente y en algunas universidades casi anecdótico. Creemos que estos resultados deberían funcionar como la base para un programa de acción de las universidades que consideran que una parte de su misión o de sus valores son la difusión del conocimiento.

\section{Palabras clave}

Redes sociales; Redes sociales digitales científicas (RSDC); Comunicación científica; Universidad española; Estudios de comunicación; Altmétricas; ResearchGate, Academia.edu. 


\begin{abstract}
Scientific social networks are a new meeting place for researchers and a major instrument for the promotion of knowledge. Their use is increasing in the scientific community, as they are practical tools to disseminate research results and to share knowledge. This paper examines the presence of 77 Spanish universities in the two main scientific social networks (ResearchGate and Academia.edu). The results show that, despite the continuous development that those networks are having worldwide, the number of Spanish members in them is considered insufficient and in some universities is almost anecdotal. These results should be the starting point for an action program of the universities to increase the membership of their academic staff in those networks, as part of their mission of disseminating knowledge.
\end{abstract}

\title{
Keywords
}

Social networks; Scientifics social networks; Spanish university; Scientific communication; Communication studies; Altmetrics; ResearchGate; Academia.edu.

González-Díaz, Cristina; Iglesias-García, Mar; Codina, Lluís (2015). "Presencia de las universidades españolas en las redes sociales digitales científicas: caso de los estudios de comunicación”. El profesional de la información, v. 24, n. 5, pp. 640-647.

http://dx.doi.org/10.3145/epi.2015.sep.12

\section{Introducción}

La web 2.0 ha supuesto una dinamización a pasos de gigante en el intercambio y difusión de información, con una enorme oferta de posibilidades y servicios. Atendiendo a Codina (2009), si nos guiamos por la percepción de la mayoría de los ciudadanos, se vincula la web 2.0 con las redes sociales. El origen de éstas es muy anterior al nacimiento de internet, pero cobran una dimensión sin precedentes con la llegada del entorno 2.0 (Martorell-Fernández, 2014). Era solamente cuestión de tiempo que estas posibilidades se trasladaran al campo de la actividad científica, dando como resultado la que se denomina ciencia 2.0, entendida como el entorno y el conjunto de aplicaciones que facilita a los científicos la gestión y búsqueda de la información, la colaboración entre profesionales y la comunicación y divulgación de los resultados para otorgarles mayor visibilidad e impacto (CabezasClavijo; Torres-Salinas; Delgado-López-Cózar, 2009; Robinson-García; Delgado-López-Cózar; Torres-Salinas, 2011).

En su fase embrionaria, la aplicación de la web 2.0 a la ciencia estuvo caracterizada por el uso de blogs. Sin embargo, son las redes sociales las que poseen un peso indiscutible en la web social. Este impacto también se ha reflejado en la actividad científica y académica con la aparición de redes sociales académicas, como ResearchGate, Academia.edu, Mendeley, My Science Work, etc., que han experimentado un gran auge en los últimos años (Martorell-Fernández; Canet-Centellas, 2013).

\section{Qué son y para qué sirven}

Estas redes no tienen una única denominación. Por ejemplo, Martorell-Fernández y Canet-Centellas (2013) las Ilaman redes sociales académicas, Arriaga-Méndez, MinorJiménez y Pérez-Cervantes, (2012) redes de investigación, y Campos-Freire (2013) redes sociales digitales científicas (RSDC), que consideramos el nombre más ajustado a nuestro objeto de estudio, y es el que adoptamos aquí.

Recientes investigaciones han abordado las RSDC (Sañudo, 2012; Martorell-Fernández; Canet-Centellas, Codina, 2014; Van-Noorden, 2014; Campos-Freire; Rivera-Rogel; Rodrí- guez, 2014) con el objetivo de estudiarlas, describirlas y caracterizarlas. Se pueden definir como plataformas de comunicación en red que posibilitan a los investigadores crear y dar a conocer un perfil académico y profesional. Este perfil es la puerta de acceso para difundir investigaciones y consultar online y/o descargar referencias y otras producciones científicas. Además, se puede establecer una lista de usuarios relacionados dentro de una o varias especializaciones científicas para compartir documentos, proyectos, colaboraciones e investigaciones (Campos-Freire, 2013). Las RSDC también pueden generar estadísticas sobre visitas a los perfiles o descargas de los trabajos, dan información sobre el seguimiento e impacto de los mismos, y muestran eventos, congresos y ofertas de empleo.

\section{Las RDSC son la puerta de acceso para difundir investigaciones y consultar onli- ne y/o descargar referencias y otras pro- ducciones científicas}

De acuerdo con Torres-Salinas y Delgado-López-Cózar (2009), la publicación científica no debe acabar con la aceptación del manuscrito, y más cuando el mismo se ha publicado en una revista que no es de acceso abierto, o que tiene poca visibilidad.

Al parecer, todavía existe cierta reticencia al uso de las RSDC por parte de la comunidad investigadora. Algunos autores siguen viendo los canales tradicionales como los más válidos (Robinson-García; Delgado-López-Cózar; Torres-Salinas, 2011) y se pone bajo sospecha cualquier tipo de evaluación que no esté sometida a la revisión por pares tradicionalmente realizada por las revistas (Torres-Salinas, 2008). Además el miedo al robo de ideas o datos, la falta de crédito o reconocimiento, la edad de los científicos (la mayoría de los investigadores principales rondan los 51 años, lejos del perfil de usuario de la web 2.0) o el tiempo para introducir los datos, hacen plantearse a algunos investigadores si este tipo de plataformas son realmente útiles (Cabezas-Clavijo; Torres-Salinas; Delgado-López-Cózar, 2009). 
Tabla 1. Número de perfiles de ResearchGate y Academia.edu en el mundo y en las universidades españolas (marzo 2015)

\begin{tabular}{|l|c|c|c|}
\hline \multicolumn{1}{|c|}{ RSDC } & $\begin{array}{c}\text { Año de } \\
\text { creación }\end{array}$ & $\begin{array}{c}\text { No perfiles } \\
\text { total }\end{array}$ & $\begin{array}{c}\text { No perfiles en universi- } \\
\text { dades españolas }\end{array}$ \\
\hline Academia.edu & 2008 & 20 millones & 68.887 \\
\hline ResearchGate & 2008 & 6 millones & 77.526 \\
\hline
\end{tabular}

A pesar de todo, las RSDC han aumentado notablemente sus miembros (tabla 1), como por ejemplo ResearchGate, que pasó de tres millones de perfiles en enero de 2014 a seis millones en enero de 2015 (ResearchGate, 2015).

Con el fin de valorar el impacto que estas redes pueden tener en la ciencia española, presentamos:

- propuesta de análisis, tomando como referencia la teoría y sistema de análisis de redes sociales (ARS) (Aguirre, 2011);

- resultados obtenidos sobre la penetración de dos importantes RSDC. Estos datos no solamente proporcionan información sobre estas redes, sino inevitablemente sobre las propias universidades, independientemente del valor que se dé a esta última información.

\section{ResearchGate y Academia.edu en las universidades españolas}

En este trabajo se estudia la presencia de las universidades españolas en ResearchGate y Academia.edu, las dos redes que tienen más miembros.

Cabe señalar que estas redes no establecen distinción por categoría profesional o ámbito laboral de sus miembros. A través de un análisis previo a este trabajo, se ha comprobado que aunque hay un claro predominio de miembros que son docentes, también existe un gran número de usuarios con otros perfiles, como estudiantes de doctorado y máster. Para establecer el número de personas que tienen la posibilidad de formar parte de las RSDC en las universidades españolas, esta investigación toma como referencia tanto profesorado como alumnado de máster. De este modo, cuando nos referimos a personal académico abarcamos tanto al personal docente e investigador (PDI) como a los estudiantes de posgrado (datos obtenidos del Ministerio de Educación, Cultura y Deporte del curso 2012/2013 para el profesorado y 2013/2014 para alumnado, últimos disponibles).

A partir de los datos extraídos de los perfiles en las redes, se observa un total de 77 universidades, 48 públicas y 29 privadas (de las 83 que enumera el Ministerio de Educación, 6 fueron descartadas por no disponer de datos).

Para un análisis detallado, se ha elaborado un ranking de las universidades, según el número de miembros en las RSDC, relacionándolo con el personal académico que pertenece a cada institución.

\section{ResearchGate y las universidades españolas}

Con más de 6 millones de miembros en enero de 2015, ResearchGate es la segunda red social científica más utilizada en el mundo, y la primera en universidades españolas, con un total de 77.526 perfiles.
La UCM, UB y UV (Estudi General) son las que poseen mayor número de perfiles en esta RSDC y también las que tienen mayor número de docentes y estudiantes de máster (tabla 3). Sin embargo, en el ranking de miembros adscritos a la universidad le sigue la USE (puesto 5 ) pero su número de usuarios en ResearchGate desciende al 9o. El caso más destacado lo representa la UNED, que tiene el mayor número de estudiantes de máster y profesores (8.885) y a pesar de ello, su número de perfiles en ResearchGate no está ni entre los 10 primeros (ocupa el puesto 19, con 1.273 perfiles).

Tabla 2. Universidades citadas. Acrónimos utilizados

\begin{tabular}{|c|c|}
\hline Universidad & Acrónimo \\
\hline Universidad a Distancia de Madrid & Udima \\
\hline Universidad Abat Oliva CEU & UAO-CEU \\
\hline Universidad Autónoma de Madrid & UAM \\
\hline Universidad Carlos III de Madrid & UC3M \\
\hline Universidad Católica San Antonio & UCAM \\
\hline Universidad Católica Sta. Teresa de Jesús de Ávila & Ucavila \\
\hline Universidad Complutense de Madrid & UCM \\
\hline Universidad de Alcalá de Henares & $U A H$ \\
\hline Universidad de Alicante & $U A$ \\
\hline Universidad de Castilla La Mancha & UCLM \\
\hline Universidad de Extremadura & UEX \\
\hline Universidad de Granada & UGR \\
\hline Universidad de La Laguna & ULL \\
\hline Universidad de La Rioja & $U R$ \\
\hline Universidad de Las Palmas de Gran Canaria & Ulpgc \\
\hline Universidad de León & ULE \\
\hline Universidad de Málaga & UMA \\
\hline Universidad de Murcia & $U M U$ \\
\hline Universidad de Navarra & UNA \\
\hline Universidad de Oviedo & Uniovi \\
\hline Universidad de Salamanca & USAL \\
\hline Universidad de Santiago de Compostela & USC \\
\hline Universidad de Sevilla & USE \\
\hline Universidad de Vigo & Uvigo \\
\hline Universidad de Zaragoza & Unizar \\
\hline Universidad del País Vasco & UPV/EHU \\
\hline Universidad Internacional de Catalunya & UIC \\
\hline Universidad Internacional de La Rioja & UNIR \\
\hline Universidad Loyola Andalucía & ULA \\
\hline Universidad Nacional de Educación a Distancia & UNED \\
\hline Universidad Pablo de Olavide & UPO \\
\hline Universidad Politécnica de Cartagena & UPCA \\
\hline Universidad Politécnica de Madrid & UPM \\
\hline Universidad Pontificia de Salamanca & UPSA \\
\hline Universidad Rey Juan Carlos & URJC \\
\hline Universidad San Pablo CEU & CEU \\
\hline Universitat Autònoma de Barcelona & $U A B$ \\
\hline Universitat de Barcelona & $U B$ \\
\hline Universitat de Girona & UGI \\
\hline Universitat de València & UV \\
\hline Universitat Jaume I de Castelló & ال ال \\
\hline Universitat Oberta de Catalunya & UOC \\
\hline Universitat Politècnica de Catalunya & UPC \\
\hline Universitat Politècnica de València & UPV \\
\hline Universitat Pompeu Fabra & UPF \\
\hline Universitat Ramon Llull & URL \\
\hline Universitat Rovira i Virgili & URV \\
\hline
\end{tabular}


Caídas tan grandes como las de la UNED también se han encontrado en otras instituciones, como es el caso de la UNIR.

Ésta ocupa el puesto 9 en número de estudiantes de máster y profesorado y, sin embargo, en el ranking en número de perfiles en ResearchGate su puesto baja hasta el 64 .

También se observa el caso contrario: universidades que están mejor posicionadas en número de perfiles de ResearchGate a pesar de tener menor número de personal académico, como la UPM, que ocupa el puesto 13 en número de adscritos a la universidad y el 8 en perfiles en ResearchGate (2.610). Ocurre lo mismo en la USC, en el puesto 18 en ranking de personal académico y en el 13 en perfiles en ResearchGate (2.087). De estos resultados se concluye que no hay una relación directa entre mayor número de personal académico y mayor presencia de usuarios en la RSDC en todas las universidades.

Tampoco se puede establecer relación directa entre mayor número de perfiles en ResearchGate y un mayor RG Score. Las cuatro universidades españolas con mayor número de perfiles en esta red social son las que tienen mayor número de $R G$ Score, pero a partir del quinto puesto no se sigue la misma pauta. Es el caso de la UPC y UPM: puestos 6 y 8 respectivamente en perfiles en ResearchGate, que descienden a 11 y 13 en $R G$ Score. El caso más llamativo lo representa de nuevo la UNED, que ocupa el lugar 19 en perfiles en $R e$ searchGate, cayendo su RG Score al 44.

También se observa el efecto contrario, ya que no por tener mayor número de perfiles en la red se obtiene un mejor $R G$ Score: la Uniovi, en el número 17 en el ranking por número de perfiles en ResearchGate y en el 14 en RG Score.

Con más de 6 millones de miembros, ResearchGate es la segunda plataforma científica más utilizada en el mundo, y la primera en las universidades españolas

Estas casuísticas vienen determinadas por la forma de obtener el $R G$ Score. Este resultado demuestra que, si la institución quiere tener un elevado índice en esta medida, lo que equivale a una mayor reputación científica, no es suficiente con tener un número elevado de perfiles en la RSDC, sino que esos miembros tienen que ser activos: colgar gran número de trabajos, participar en los debates, etc.

Al margen del lugar que ocupen las universidades en relación con el número de perfiles en ResearchGate y número

Tabla 3. Ranking de las 20 primeras universidades ordenadas por 1) número de miembros (PDI y estudiantes máster); 2) número de perfiles en ResearchGate; 3) $R G$ Score y 4) porcentaje extraído en función de miembros de la universidad y perfiles en esta RSDC. La métrica RG Score indica la reputación científica en función de cómo la investigación de un usuario es recibida por el resto de compañeros de la red social atendiendo a las contribuciones realizadas, las interacciones y la reputación obtenida investigador a investigador. ResearchGate calcula el RG Score de una institución en base al RG Score individual de perfiles adscritos a la misma (ResearchGate, 2015).

\begin{tabular}{|c|c|c|c|c|c|c|c|}
\hline \multicolumn{8}{|l|}{ ResearchGate } \\
\hline Universidad & $\begin{array}{l}\text { Miembros } \\
\text { universidad }\end{array}$ & Universidad & $\begin{array}{c}\text { Total } \\
\text { perfiles } R G\end{array}$ & Universidad & RG Score & Universidad & $\begin{array}{l}\text { \% perfiles RSDC en } \\
\text { función miembros } \\
\text { universidad }\end{array}$ \\
\hline UCM & 11.227 & UCM & 4.274 & $U B$ & $41.509,01$ & UPC & 59,83 \\
\hline$U B$ & 9.403 & $U B$ & 3.918 & UCM & $39.289,69$ & UPM & 54,03 \\
\hline UNED & 8.885 & UV & 3.386 & UV & $33.076,91$ & USC & 49,82 \\
\hline UV & 8.680 & UGR & 3.263 & $U G R$ & $30.098,15$ & UAM & 49,57 \\
\hline USE & 8.189 & $U A B$ & 3.189 & UPV/EHU & $29.091,90$ & $U A B$ & 49,55 \\
\hline UPV/EHU & 7.049 & UPC & 2.807 & $U A B$ & $28.786,51$ & ULA & 48,43 \\
\hline$U G R$ & 6.905 & UPV/EHU & 2.698 & USE & $25.829,01$ & ULE & 47,68 \\
\hline$U A B$ & 6.436 & UPM & 2.610 & UAM & $25.375,36$ & URV & 47,27 \\
\hline UNIR & 5.730 & USE & 2.510 & Unizar & $25.119,44$ & UGR & 47,26 \\
\hline UPV & 5.402 & UPV & 2.490 & USC & $24.442,17$ & UGI & 46,81 \\
\hline Unizar & 4.942 & UAM & 2.423 & UPC & $24.386,61$ & UPCA & 46,14 \\
\hline UAM & 4.888 & Unizar & 2.192 & UPV & $22.468,39$ & UPV & 46,09 \\
\hline UPM & 4.831 & USC & 2.087 & UPM & $20.033,42$ & ULL & 45,86 \\
\hline UMU & 4.820 & $U M U$ & 1.681 & Uniovi & $15.897,07$ & $U R$ & 45,13 \\
\hline UPC & 4.692 & UMA & 1.582 & UMU & $15.662,76$ & Unizar & 44,35 \\
\hline URJC & 4.320 & USAL & 1.577 & $U M A$ & $14.584,31$ & Uniovi & 44,21 \\
\hline UMA & 4.303 & Uniovi & 1.448 & USAL & $14.193,46$ & UIC & 42,72 \\
\hline USC & 4.189 & $U A$ & 1.426 & Uvigo & $13.653,35$ & USAL & 41,70 \\
\hline Udima & 3.808 & UNED & 1.273 & UNA & $13.143,91$ & $U B$ & 41,67 \\
\hline USAL & 3.782 & Uvigo & 1.258 & UCLM & $12.958,11$ & Ulpgc & 41,65 \\
\hline
\end{tabular}


de miembros adscritos a cada centro, en términos porcentuales es donde encontramos las mayores discrepancias. Cuando se examina el porcentaje de perfiles en la red social con relación al número de profesores y estudiantes de máster en su conjunto, se observa que no son las instituciones de mayor envergadura las que se encuentran en las primeras posiciones. Encabezan la lista la UPM, UPC y USC, que no están ni entre los 5 primeros puestos en el ranking de perfiles de ResearchGate, ni entre los 10 primeros en personal académico. Cabe destacar el caso de la URV o la ULA. Esta última ocupa el lugar 74 en número de profesores y estudiantes y el 68 en perfiles en ResearchGate. Es decir, hay centros que a pesar de tener un menor volumen de personal académico, tienen porcentualmente más presencia en esta RSDC que aquellos de mayor envergadura dentro del panorama estatal.

\section{La penetración de ResearchGate y} Academia.edu en las universidades españolas es todavía escasa y en algunas universidades casi anecdótica

Sin embargo, el dato general y más sorprendente es que 10 de las universidades estudiadas no llegan ni al $10 \%$ de penetración y aquellas con índices mayores sólo bordean o superan tímidamente el $50 \%$. Estos datos muestran la exigua presencia de investigadores españoles en ResearchGate, teniendo en cuenta que esta RSDC fue fundada en 2008 y que su versión en castellano lleva operativa ya 4 años.

\section{Academia.edu y las universidades españolas}

A pesar de ser Academia.edu la RSDC más popular, con 20 millones de perfiles en todo el mundo, entre las universidades españolas ocupa el segundo lugar, con 77.126 perfiles.

Al igual que en ResearchGate, no puede establecerse de forma generalizada que las universidades españolas con mayor número de personal académico tengan a su vez mayor número de perfiles en Academia.edu. Hay centros como la $U C M, U B$ o UV en los que se observa esa relación, aunque no de forma exacta en el ranking. Sin embargo, esta investigación muestra que hay universidades con gran envergadura en personal académico, como la UPV/EHU y UPV, que no se corresponden con los primeros puestos en número de perfiles en Academia.edu, ya que su presencia en la misma desciende considerablemente. También se observa la situación inversa, como en la USAL, UA o UOC.

En porcentajes del número de perfiles en Academia.edu en función del número de personal académico encabezan este ranking la UPSA y UAO-CEU, universidades que se encuentran en los últimos puestos en lo que a personal académico se refiere (la UPSA ocupa el puesto 72 , mientras que la UAO ocupa el último lugar). Cabe destacar, también de estas dos instituciones, que superan el $100 \%$, lo que indica que si bien en esta investigación se ha tenido en cuenta el número de PDI y estudiantes de máster, con este dato se demuestra que existe otro tipo de perfiles, posiblemente de alumnos que están cursando los últimos años de grado, que también hacen uso de esta RSDC, aspecto a contemplar en futuras investigaciones. También podría deberse a la duplicidad de perfiles encontrados, especialmente en esta RSDC.

Tabla 4. Ranking de las 20 primeras universidades ordenadas por 1) número de miembros (PDI y estudiantes máster); 2) número de perfiles en Academia. edu y 3) porcentaje extraído en función de miembros de la universidad y perfiles en esta RSDC

\begin{tabular}{|c|c|c|c|c|c|}
\hline \multicolumn{2}{|c|}{ Academia.edu } & \multirow[b]{2}{*}{ Universidad } & \multirow[b]{2}{*}{$\begin{array}{l}\text { Total perfiles } \\
\text { Academia.edu }\end{array}$} & \multirow[b]{2}{*}{ Universidad } & \multirow[b]{2}{*}{$\begin{array}{c}\text { \% perfiles RSDC en función } \\
\text { miembros universidad }\end{array}$} \\
\hline Universidad & $\begin{array}{l}\text { Miembros } \\
\text { universidad }\end{array}$ & & & & \\
\hline UCM & 11.227 & UCM & 4.833 & UPSA & 124,13 \\
\hline$U B$ & 9.403 & $U A B$ & 3.991 & UAO-CEU & 103,88 \\
\hline UNED & 8.885 & $U B$ & 3.775 & UAM & 63,48 \\
\hline UV & 8.680 & UAM & 3.103 & $U A B$ & 62,01 \\
\hline USE & 8.189 & UV & 3.002 & $U A$ & 45,15 \\
\hline UPV/EHU & 7.049 & UNED & 2.883 & UC3M & 44,72 \\
\hline UGR & 6.905 & USE & 2.517 & USAL & 43,89 \\
\hline$U A B$ & 6.436 & UGR & 1.820 & UCM & 43,05 \\
\hline UNIR & 5.730 & UPM & 1.812 & Ucavila & 42,21 \\
\hline UPV & 5.402 & UMA & 1.709 & UOC & 42,11 \\
\hline Unizar & 4.942 & USAL & 1.660 & $U B$ & 40,15 \\
\hline UAM & 4.888 & $U A$ & 1.601 & ULL & 39,84 \\
\hline UPM & 4.831 & UC3M & 1.587 & UMA & 39,72 \\
\hline UMU & 4.820 & UOC & 1.562 & Uniovi & 38,44 \\
\hline UPC & 4.692 & Unizar & 1.562 & URV & 38,32 \\
\hline URJC & 4.320 & UPC & 1.552 & UPO & 38,21 \\
\hline UMA & 4.303 & UPV/EHU & 1.441 & ULA & 37,74 \\
\hline USC & 4.189 & USC & 1.426 & UPM & 37,51 \\
\hline Udima & 3.808 & UPF & 1.314 & Ulpgc & 36,11 \\
\hline USAL & 3.782 & UMU & 1.308 & UPF & 34,94 \\
\hline
\end{tabular}


Tabla 5. Ranking de las 20 primeras universidades ordenadas por número de perfiles en ResearchGate y Academia.edu en total y en el ámbito de la comunicación.

\begin{tabular}{|c|c|c|c|c|c|c|c|}
\hline \multicolumn{4}{|c|}{ ResearchGate } & \multicolumn{4}{|c|}{ Academia.edu } \\
\hline Universidad & $\begin{array}{c}\text { Total } \\
\text { perfiles }\end{array}$ & Universidad & $\begin{array}{c}\text { Perfiles de } \\
\text { comunicación }\end{array}$ & Universidad & $\begin{array}{c}\text { Total } \\
\text { perfiles }\end{array}$ & Universidad & $\begin{array}{l}\text { Perfiles de } \\
\text { comunicación }\end{array}$ \\
\hline UCM & 4.274 & UPF & 99 & UCM & 4.833 & UCM & 473 \\
\hline$U B$ & 3.918 & UCM & 97 & $U A B$ & 3.991 & $U A B$ & 284 \\
\hline UV & 3.386 & $U A B$ & 74 & $U B$ & 3.775 & URJC & 280 \\
\hline UGR & 3.263 & URL & 50 & UV & 3.002 & USE & 183 \\
\hline$U A B$ & 3.189 & URJC & 49 & USE & 2.517 & UC3M & 151 \\
\hline UPV/EHU & 2.698 & UOC & 48 & UGR & 1.820 & URL & 144 \\
\hline USE & 2.510 & UMA & 43 & UMA & 1.709 & UMA & 140 \\
\hline UPV & 2.490 & CEU & 42 & USAL & 1.660 & UV & 120 \\
\hline Unizar & 2.192 & $U A$ & 36 & $U A$ & 1.601 & 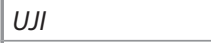 & 102 \\
\hline USC & 2.087 & USAL & 34 & UC3M & 1.587 & UPF & 85 \\
\hline UMU & 1.681 & USC & 31 & UOC & 1.562 & UNA & 81 \\
\hline UMA & 1.582 & UPV/EHU & 30 & Unizar & 1.562 & USC & 79 \\
\hline USAL & 1.577 & UGI & 29 & UPV/EHU & 1.441 & Uvigo & 71 \\
\hline$U A$ & 1.426 & UGR & 26 & USC & 1.426 & UOC & 70 \\
\hline Uvigo & 1.258 & UC3M & 24 & UPF & 1.314 & CEU & 68 \\
\hline URV & 1.236 & $U A H$ & 23 & UMU & 1.308 & UPV/EHU & 67 \\
\hline UC3M & 1.231 & U & 23 & URJC & 1.264 & URV & 59 \\
\hline UCLM & 1.199 & UPV & 22 & UPV & 1.199 & UPSA & 58 \\
\hline UNA & 1.141 & UV & 22 & UCLM & 1.021 & USAL & 52 \\
\hline UPF & 1.126 & UEX & 20 & URV & 1.002 & UCAM & 47 \\
\hline
\end{tabular}

A excepción de la UAM o UAB, también lideran este ranking universidades como la UC3M o UA, que no se encuentran ni entre las 20 primeras en número de personal académico.

Sin contar con los porcentajes de las dos primeras universidades que lideran este ranking, observamos de forma genérica unos porcentajes algo mayores de penetración en esta RSDC que los hallados en ResearchGate. Sin embargo, el $18 \%$ de las instituciones estudiadas (14) tienen un porcentaje de penetración que no supera el $10 \%$, demostrando también la escasa presencia de Academia.edu en la comunidad universitaria española, teniendo en cuenta que lleva operativa más de 7 años.

\section{Miembros adscritos a estudios de comunicación}

Si bien todos los miembros de la comunidad universitaria deben dar visibilidad a sus trabajos académicos, se podría presuponer que aquellos adscritos a estudios de comunicación deberían tener una presencia mayor en las RSDC.

Por este motivo, atendiendo a la formación de los autores del estudio y a las peculiares características de los investigadores en el área de comunicación, también se ha observado la presencia de perfiles en las RSDC atendiendo a las universidades que poseen estos estudios. De las 77 que enmarcan esta investigación, 54 poseen estudios en comunicación, y todas utilizan ambas RSDC, exceptuando la Universidad Europea de Canarias (que no está presente en ninguna).

Aunque en el conjunto de universidades españolas existe una mayor presencia de perfiles en ResearchGate, en el ámbito de comunicación la pauta se invierte, y se muestra una clara preferencia por Academia.edu. Este dato coincide con estudios realizados por Campos-Freire, Rivera-Rogel y Rodríguez (2014) y Punín, Direito-Rebollal y Calva (2014), que confirman que a nivel global, en ResearchGate predominan perfiles de ciencias naturales y aplicadas, mientras que en Academia.edu son mayoritarios los investigadores de ciencias sociales.

La tabla 5 establece un ranking de las 20 universidades que mayor número de perfiles tienen en ambas RSDC en su totalidad y específicamente en comunicación.

Hay un mayor número perfiles en Academia.edu entre los miembros adscritos a estudios de comunicación

La UCM, UAB, UV, USE y UB lideran los rankings en ambas RSDC, en lo que a número total de perfiles se refiere. Sin embargo, atendiendo al ranking de personal adscrito a estudios de comunicación se encuentran excepciones.

En ResearchGate destaca el caso de la UPF, que lidera el número de perfiles en comunicación y ocupa el puesto 20 en el ranking de usuarios total de esta RSDC, con 99 perfiles. Otro caso es el de la UOC, que en el ranking de perfiles total ocupa el puesto 29 , y en el de comunicación asciende hasta el sexto lugar (48 perfiles).

En Academia.edu esta situación se repite en la URJC, que ocupa el puesto 3 en comunicación (280 perfiles), y en usuarios totales desciende al 17. Más pronunciado es el caso de la $U R L$, que ocupa el puesto 34 , y sube al número 6 en comunicación (144 perfiles). 


\section{Conclusiones y discusión}

A pesar del avance continuo que están experimentando las RSDC en todo el mundo, su penetración en las universidades españolas es todavía escasa (bordeando o rebasando tímidamente el $50 \%$ de penetración en las mejor posicionadas) y en algunas universidades es casi anecdótica, no superando el $10 \%$ del personal académico. Estos datos revelan que en las universidades españolas la presencia de las RSDC está todavía en una fase inicial, si tenemos en cuenta que uno de los pilares en los que se fundamenta la ciencia es su difusión, y que las RSDC estudiadas (que llevan más de siete años en marcha) son un claro instrumento para ello.

Esto podría ser debido a dos supuestos:

- estas redes todavía no son suficientemente conocidas;

- quienes las conocen, no consideran que sean útiles, para el tiempo que requiere crear y mantener un perfil actualizado.

Este segundo supuesto se podría amparar en la pregunta de si realmente son útiles las RSDC. La utilidad real y las ventajas que supone tener un perfil en ellas es actualmente objeto de estudio, y ciertamente está por determinar. En este sentido señalamos la particularidad de ResearchGate y su indicador de reputación ( $R G$ Score), que Academia.edu no posee. Mientras que el índice $H$ de Google mide impacto y productividad, el $R G$ Score registra impacto y popularidad, es decir, producción e interacción del investigador ante sus colegas (Punín; Direito-Rebollal; Calva, 2014). Tal y como señalan Campos-Freire, Rivera-Rogel y Rodríguez (2014), a diferencia de las métricas tradicionales, el $R G$ Score se centra en el investigador y es mucho más dinámico, porque a través de la conversación y la interacción científica se va autogenerando constantemente. Bajo este contexto, de forma al parecer imparable se van consolidando las altmétricas (o métricas alternativas), una serie de indicadores que miden el impacto de la investigación, cuantificando su presencia en la web social (Galligan; Dyas-Correia, 2013; Thelwall et al., 2013; Borrego, 2014; Robinson-García et al., 2014), como herramienta complementaria al método tradicional de citas en los $J C R(I S I)$ y $S J R$.

La implementación de las altmétricas en la reputación de la investigación puede suponer la clave para incentivar y consolidar el uso de las RSDC, y más si en los próximos años, agencias evaluadoras como Aneca o Cneai añaden altmétricas entre sus parámetros de calidad de impacto de la productividad científica.

Todos estos indicios, muy solventes, nos estarían indicando que las universidades españolas pueden estar perdiendo oportunidades en este ámbito si sus responsables, o los propios investigadores no son conscientes de ello. Por tanto, entendemos que los resultados, además de ser una exploración de una situación determinada, podrían o incluso tal vez deberían, tomarse como un programa para la acción por parte de las universidades que hacen suyo el valor según el cual una de sus misiones es la difusión del conocimiento. Por no mencionar los motivos de ambición (en este caso ambición muy legítima) de promoción de sus investigadores.

Esta investigación muestra que ResearchGate es la RSDC que más perfiles tiene en las universidades españolas, si bien Academia.edu tiene un mayor número de perfiles en todo el mundo. Al mismo tiempo, se observa que hay un mayor número perfiles en Academia.edu entre los miembros adscritos a estudios de comunicación, posiblemente debido a que se considera más especializada en ciencias sociales (Punín; Direito-Rebollal; Calva, 2014).

Finalmente se observa que un mayor número de personal académico en las universidades no se traduce en un mayor número de perfiles en las RSDC. En el caso de ResearchGate, que también establece un índice de reputación científica ( $R G$ Score), un mayor numero de perfiles tampoco asegura un mejor índice en este indicador, ya que para calcularlo no es suficiente con crear perfiles, sino que éstos tienen que ser activos.

No puede establecerse de forma generalizada que las universidades españolas con mayor número de personal académico tengan a su vez mayor número de perfiles en las RDSC

Esta investigación no ha estado exenta de limitaciones. Señalamos la imposibilidad de comparar datos de ResearchGate y Academia.edu más allá del número de perfiles, pues no ofrecen los mismos indicadores:

- ResearchGate computa el RG Score, número de publicaciones y puntos totales por perfiles adscritos a una universidad, así como los puntos globales adscritos a una disciplina de conocimiento concreto (en este caso comunicación).

- Academia.edu sólo ofrece el número de publicaciones adscritas a una determinada disciplina, no la totalidad.

Esto, en realidad, también debería ser motivo de un programa de acción: nuevas investigaciones que impulsen la estandarización de estas métricas.

Por otro lado, se observa en algunos casos la duplicidad de perfiles y de departamentos (en especial en Academia.edu) con diferentes nombres y en diferentes idiomas, preferentemente inglés y castellano. Este error debería ser tenido en cuenta por las RSDC para depurar el número real de perfiles y departamentos. Además de la duplicidad de perfiles, se ha observado la diversidad del tipo de personal académico. Si bien en su mayoría son docentes que utilizan las RSDC para la difusión de sus trabajos o investigaciones en curso, también se detecta un número considerable de perfiles de estudiantes de doctorado y máster.

Investigaciones futuras deberían contemplar este hecho para determinar y describir los perfiles de estas RSDC y, dependiendo del perfil, cuál es su verdadero uso y no presuponer que únicamente los investigadores/docentes hacen uso de las mismas.

En todo caso, esta investigación aporta elementos para un programa de acción por parte de las universidades que consideren que forma parte de su misión la promoción y la difusión del conocimiento, y por otro lado, para nuevas investigaciones que luchen para paliar los problemas de comparar los indicadores señalados. 


\section{Nota}

Este trabajo forma parte del proyecto Audiencias activas y periodismo. Interactividad, integración en la web y buscabilidad de la información periodística: CSO2012-39518-C04-02. Plan Nacional de I+D+i, Ministerio de Economía y Competitividad (España).

\section{Bibliografía}

Aguirre, Julio-Leónidas (2011). Introducción al análisis de redes sociales. Buenos Aires: Documentos de trabajo, 82, Centro Interdisciplinario para el Estudio de Políticas Públicas.

Arriaga-Méndez, Juana; Minor-Jiménez, Marcos G.; PérezCervantes, Mónica-Luz (2012). "Retos y desafíos de las redes de investigación". Revista iberoamericana sobre calidad, eficacia y cambio en educación, v. 10, n. 3 p. 177-183. http://www.redalyc.org/pdf/551/55124665015.pdf

Borrego, Ángel (2014). "Altmétricas para la evaluación de la investigación y el análisis de necesidades de información". El profesional de la información, v. 23, n. 4, pp. 352-357. http://www.elprofesionaldelainformacion.com/contenidos/2014/ jul/02.pdf http://dx.doi.org/10.3145/epi.2014.jul.02

Cabezas-Clavijo, Álvaro; Torres-Salinas, Daniel; DelgadoLópez-Cózar, Emilio (2009). "Ciencia 2.0: catálogo de herramientas e implicaciones para la actividad investigadora". El profesional de la información, v. 18, n. 1, pp. 72-79.

http://www.elprofesionaldelainformacion.com/contenidos/2009/ enero/09.pdf

http://dx.doi.org/10.3145/epi.2009.ene.10

Campos-Freire, Francisco (2013). "Introducción. La investigación y gestión de las redes sociales digitales". En: CamposFreire, Francisco (ed.). Investigación y gestión de las redes digitales. Cuadernos artesanos de comunicación, 50. La Laguna (Tenerife): Sociedad Latina de Comunicación Social, pp. 7-53. ISBN: 9788415698425

http://www.cuadernosartesanos.org/cac50.pdf

Campos-Freire, Francisco; Rivera-Rogel, Diana; Rodríguez, Claudia (2014). "La presencia e impacto de las universidades de los países andinos en las redes sociales digitales". Revista latina de comunicación social, n. 69, pp. 571- 592.

http://dx.doi.org/10.4185/RLCS-2014-1025

Codina, Lluís (2009). “Ciencia 2.0: redes sociales y aplicaciones online para académicos". Hipertext.net, n. 7.

http://www.upf.edu/hipertextnet/numero-7/ciencia-2-0.html

Galligan, Finbar; Dyas-Correia, Sharon (2013). "Altmetrics: rethinking the way we measure". Serials review, v. 39, n. 1, pp. 56-61. http://dx.doi.org/10.1016/j.serrev.2013.01.003

Martorell-Fernández, Sandra (2014). Investigación académica y web social: Análisis y propuestas en el campo de los estudios filmicos. Tesis doctoral. Universitat Politècnica de València.

Martorell-Fernández, Sandra; Canet-Centellas, Fernando
(2013). "Investigar desde internet: Las redes sociales como abertura al cambio". Historia y comunicación social, v. 18, n. especial, pp. 663-675.

http://revistas.ucm.es/index.php/HICS/article/viewFile/44276/41834 http://dx.doi.org/10.5209/rev_HICS.2013.v18.44276

Martorell-Fernández, Sandra; Canet-Centellas, Fernando; Codina, Lluís (2014). "Canalizar audiencias académicas: Propuesta de una red social para investigadores en estudios fílmicos". Hipertext.net, n. 12.

http://raco.cat/index.php/Hipertext/article/view/274217

http://dx.doi.org/10.2436/20.8050.01.11

Punín, María-Isabel; Direito-Rebollal Sabela; Calva, Daniela (2014). "La participación e interacción de los investigadores de comunicación de países iberoamericanos en las redes sociales digitales científicas". En: VI Congreso internacional Latina de comunicación social, pp. 1-33.

http://www.revistalatinacs.org/14SLCS/2014_actas/119_Punin.pdf

ResearchGate (2015). Celebrating 6 million members.

https://explore.researchgate.net/display/news/2015/01/21/ Celebrating+6+million+members

Robinson-García, Nicolás; Delgado-López-Cózar, Emilio; Torres-Salinas, Daniel (2011). "Cómo comunicar y diseminar información científica en internet para obtener mayor visibilidad e impacto". Aula abierta, v. 39, n. 3, pp. 41-50. http://digibug.ugr.es/handle/10481/19199\#.VcRxQOjt/Bc

Robinson-García, Nicolás; Torres-Salinas, Daniel; Zahedi, Zohreh; Costas, Rodrigo (2014). "New data, new possibilities: Exploring the insides of Altmetric.com". El profesional de la información, July-August, v. 23, n. 4, pp. 359-366. http://dx.doi.org/10.3145/epi.2014.jul.03

Sañudo, Lya (2012). "El papel de las redes profesionales de investigación en un mundo globalizado". Revista iberoamericana sobre calidad, eficacia y cambio en educación, v. 10, n. 3, pp. 136-143.

http://www.redalyc.org/pdf/551/55124665011.pdf

Thelwall, Mike; Haustein, Stefanie; Larivière, Vincent; Sugimoto, Cassidy (2013). "Do altmetrics work? Twitter and ten other social web services". PloS one, v. 8, n. 5. http://dx.doi.org/10.1371/journal.pone.0064841

Torres-Salinas, Daniel (2008). “El paradigma 2.0 en las grandes revistas científicas". En: $3^{\text {rd }}$ intI LIS-EPI meeting. Innovación en información. Valencia, 24-25 septiembre, pp. 24-25. http://eprints.rclis.org/14080

Torres-Salinas, Daniel; Delgado-López-Cózar, Emilio (2009). "Estrategias para mejorar la difusión de los resultados de investigación con la web 2.0". El profesional de la información, v. 18, n. 5 , pp. 534-539.

http://eprints.rclis.org/13901

http://dx.doi.org/10.3145/epi.2009.sep.07

Van-Noorden, Robert (2014). “Online collaboration: Scientists and the social network". Nature news, v. 512, n. 7513, pp. 126-129.

http://dx.doi.org/10.1038/512126a 